\title{
Surgical lung biopsy in onco-hematological patients with diffuse pulmonary infiltrates and mechanical ventilation in the ICU
}

\author{
EDUARDO MANTOVANI CARDOSO ${ }^{1}$, ALEXANDRE GALVÃO BUENO ${ }^{2}$, DANIEL AUGUSTO PAVAN ${ }^{3}$, \\ ADEMAR DANTAS CUNHA Jr. ${ }^{4}$, RAYSA CRISTINA SCHMIDT ${ }^{1}$ and PÉRICLES A.D. DUARTE ${ }^{1}$ \\ Departments of ${ }^{1}$ Critical Care Medicine, ${ }^{2}$ Pathology, ${ }^{3}$ Thoracic Surgery and ${ }^{4}$ Oncology, \\ Hospital do Câncer/UOPECCAN, Cascavel, Paraná 85806-300, Brazil
}

Received October 2, 2018; Accepted February 14, 2019

DOI: $10.3892 / 01.2019 .10065$

\begin{abstract}
Among onco-hematological patients with acute respiratory failure (ARpF), surgical lung biopsy (SLB) could contribute to the medical management, by guiding initiation, maintenance or discontinuation of diagnostic and therapeutic interventions. The aim of the present study was to evaluate the results of SLB in these patients in an oncological center from a medium-income country, as well as analyze if this procedure is clinically useful in this context, and its impact on complications and mortality. This observational retrospective study analyzed onco-hematological patients with $\mathrm{ARpF}$ in the Intensive Care Unit (ICU) of a cancer center in southern Brazil between 2010-2016, who required mechanical ventilation and were submitted to open SLB. Among the studied population $(\mathrm{n}=17)$, the most commonly found etiology was infectious, present in $\sim 50 \%$ of the biopsies, followed by unspecific inflammatory infiltrate acute respiratory distress syndrome and interstitial fibrosis, alveolar hemorrhage, neoplastic infiltrate and pulmonary embolism. Biopsy has led to a change of management in $63.3 \%$ of patients that were alive when results were available; however, $35 \%$ of patients succumbed prior to the pathological result. There was no requirement for re-operation or mortality attributable to the procedure. However, ICU mortality was elevated (88\%). SLB in onco-hematological
\end{abstract}

Correspondence to: Dr Péricles A.D. Duarte, Department of Critical Care Medicine, Hospital do Câncer/UOPECCAN, R. Itaquatiaras, 769 Cascavel, Paraná 85806-300, Brazil

E-mail: pericles.duarte@uol.com.br

Abbreviations: APACHE, acute physiology and chronic health evaluation score; ARDS, acute respiratory distress syndrome; ARnF, acute renal failure; $\mathrm{ARpF}$, acute respiratory failure; $\mathrm{BMI}$, body mass index; BMT, bone marrow transplant; BOOP, bronchiolitis obliterans/organizing pneumonia; $\mathrm{CHF}$, congestive heart failure; ICU, intensive care unit; $\mathrm{MV}$, mechanical ventilation; $\mathrm{PaO}_{2}$, pressure of arterial oxygen; PEEP, positive end-expiratory pressure; SLB, surgical lung biopsy; SD, standard deviation

Key words: hematology, neoplasms, biopsy, thoracic surgery, respiratory insufficiency, respiration, artificial, intensive care units patients mechanically ventilated in the ICU is a safe procedure with few severe complications and that contributes for diagnosis and management in the majority of cases. Due to the high mortality of this population, controlled trials may be required to establish its benefit in mortality and ICU outcomes.

\section{Introduction}

Associated to an increase in survival of cancer patients (1), in recent years it has been shown a reduction in mortality of oncological patients in the Intensive Care Unit (ICU) $(2,3)$. Those patients, however, occupy $15 \%$ of all ICU beds, with significant costs $(4,5)$. In the sub-population of onco-hematological patients, Acute Respiratory Failure (ARpF) is a common cause of ICU admission $(6,7)$ and the strongest risk factor for mortality (8). The economic impact of $\mathrm{ARpF}$, especially mechanical ventilation (MV), is enormous: Mean costs of hospitalization in patients with MV for more than 4 days exceeds US\$60,000 and, in prolonged VM (>21 days), $\$ 200,000$. Those expenses make up for $12 \%$ of hospital costs, in a total of $\$ 27$ billion dollars in the USA alone (9).

Common causes of $\mathrm{ARpF}$ in patients with hematological malignancy are infections (bacterial and opportunistic), acute pulmonary edema, alveolar hemorrhage, neoplastic infiltrate, chemotherapy adverse reactions, specific and unspecific inflammatory diseases, among other uncommon pathologies $(10,11)$.

In these patients, surgical lung biopsy (SLB) can be performed when etiology has not been established by less invasive tests (cultures, serologies) and procedures [bronchoalveolar lavage (BAL), transbronchial biopsy] (12).

The aim of the present study was to evaluate open SLB as a diagnostic strategy in onco-hematological patients with $\mathrm{ARpF}$ without defined etiology, in MV, at the ICU. Specifically, we seek to understand what is the impact of the biopsy in medical management, what are the etiologies commonly found and what are the complications and outcomes of this group of patients submitted to this intervention.

\section{Materials and methods}

Study design and setting. Observational, retrospective study, based on analysis of databases, physical charts and electronic 
medical records of patients admitted to the ICU of a dedicated oncology hospital in southern Brazil. It is an 8 bed general ICU (medical and surgical) that assists almost exclusively oncological patients.

Patients admitted to the ICU between 2010 and 2016 were evaluated. Through databases, we selected all onco-hematological patients with $\mathrm{ARpF}$ and submitted to open SLB. We analyzed also physical charts to get further information regarding the realization of SLB, treatment plans before and after the biopsy, histopathology results, complications, and outcomes. The same pathologist (AGB) that performed the histological studies revised the microscope slides for this study.

Patients. Patients with onco-hematological disorders admitted to the general ICU from 2010 to 2016 with bilateral pulmonary infiltrates and $\mathrm{ARpF}$, receiving $\mathrm{MV}$, that underwent SLB during ICU stay. There were no exclusion criteria, except 2 patients whose data from physical charting and electronic medical records were scarce and insufficient. Based on these inclusion criteria, we analyze electronic medical records and paper (physical) charting of all 17 that met the criteria.

Definitions and variables. Onco-hematological disorders: Leukemias, lymphomas, multiple myeloma, bone marrow aplasia and chronic lymphoproliferative disorders.

ARpF: Was defined clinically by the assistant ICU team.

Acute Respiratory Distress Syndrome (ARDS): Was defined according to the Berlin Consensus Conference (13).

Acute Renal Failure (ARnF): Was defined according to the AKIN criteria (14).

Sepsis: Was defined according to Sepsis-3 criteria (15).

The diagnoses of comorbidities were made by the ICU and onco-hematology healthcare team, without pre-selected criteria for the study.

During ICU stay, the patients were treated according to the ICU's own protocols regarding mechanical ventilation and weaning, sedation, nutrition, as well as management of specific conditions such as infections, sepsis and choosing antibiotics.

SLB: Open technique was the only one performed at our study, using mini-thoracotomy access, done at the bedside or the operating room. It was performed by 2 thoracic surgeons and 1 oncological surgeon experienced in the procedure. The procedure, indications and their routines remained unchanged throughout the period relating to the collection of the study.

Statistical methods. A descriptive statistical analysis was performed and percentages expressed as frequency, mean and standard deviation. Data were compared using the Chi-square test and Statistica ${ }^{\circledR} 7.0$ software (StatSoft, Inc., Tulsa, OK, USA). $\mathrm{P}<0.05$ was considered to indicate a statistically significant difference.

Ethical approval and Consent to participate. This study was conducted in accordance with the recommendations of Resolution 466/2012 of the Brazilian National Health Council. The project was approved by UNIOESTE (Western Paraná State University)'s Committee on Ethics in Research involving human beings. Due to the retrospective nature of this study the requirement for patient or the families' written informed consent was waived.

\section{Results}

The majority of the 17 patients submitted to SLB were young adults (mean: 33.8 years old- $\min =12$; $\max =58$ ) men (70.6\%), without comorbidities $(70.5 \%)$ and diagnosed with leukemia $(53 \%)$ or lymphoma $(41 \%)$. Most of them $(88 \%)$ were submitted to chemotherapy recently. ICU admission cause was medical in all patients (no surgical cases), with mean MV time before biopsy of 2 days and total MV time of about 7 days. Immediately before the biopsy, these patients were being ventilated with high PEEPs (mean: $11 \mathrm{cmH}_{2} \mathrm{O}$ ), frequently lightly thrombocytopenic (mean $99 \times 10^{3} / \mathrm{mm}^{3}$ ), using vasoactive drugs and receiving several antibiotics (88\% with 3 or more) with frequent covering of opportunistic infections-any antifungal in $70.5 \%$ of patients, antiviral in $41 \%$ or Trimethoprim-sulfamethoxazole in $41.2 \%$. Most common comorbidities were Systemic Hypertension and Diabetes Mellitus (Table I).

The most commonly found etiology on histopathology was infectious (52.3\%)-bacterial infections, Cytomegalovirus (CMV) pneumonitis, and Pneumocystis jirovecii pneumonia accounted for $17.6 \%$ of findings each. Unspecific inflammatory conditions were the second most common finding (ARDS and unspecific infiltrate), present in $29.5 \%$ of biopsies. Alveolar hemorrhage was shown in 3 biopsies (17.6\%), neoplastic infiltrate in $2(12 \%)$ and Pulmonary Embolism in 1 (6\%) (Table II, and Figs. 1-5).

There were only minor complications related to the procedure, but no major or significative early complications (Table III).

Therapeutic modifications occurred in $41 \%$ of patients. However, it has to be considered that $35.3 \%$ patients died before the histology results were available. Thus, there were therapeutic modifications in $63.3 \%$ of patients whose biopsy's results were available before death. The most common therapeutic impact was changing in antibiotics-starting or stopping antibiotics (1 patient each), antivirals ( 2 patients) and antifungals (1 patient). In one patient it was started anticoagulation and in another chemotherapy was discontinued (Fig. 1).

The mean ICU length of stay was of 9.5 days, with high ICU and in-hospital mortality (88.2\%). Approximately 1/3 (35.3\%) of patients died before the availability of biopsy results.

\section{Discussion}

The indication for SLB in onco-hematological patients with $\mathrm{ARpF}$ is limited, according to the literature. Patients with $\mathrm{ARpF}$ of unknown etiology after less invasive tests, with clinical deterioration and BAL that is inconclusive, with low diagnostic yield or high risk of complications, are generally the circumstances in which this procedure is indicated by the literature $(12,16-18)$.

In this present study, lung biopsy has evidenced a specific diagnosis in $70.5 \%$ of patients, similar to the $60-65 \%$ of previous studies (16-20). Infection was the most common finding (about $70 \%$ of biopsies), despite previous studies showing unspecific infiltrates (fibrosis, interstitial pneumonitis, ARDS) as the most prevalent biopsy finding, even though infections were still common (16-18). Among infections, the opportunistic ones were the most commonly found (52.8\%) (16-20). 
Table I. Characteristics and clinical course of patients submitted to SLB $(n=17)$.

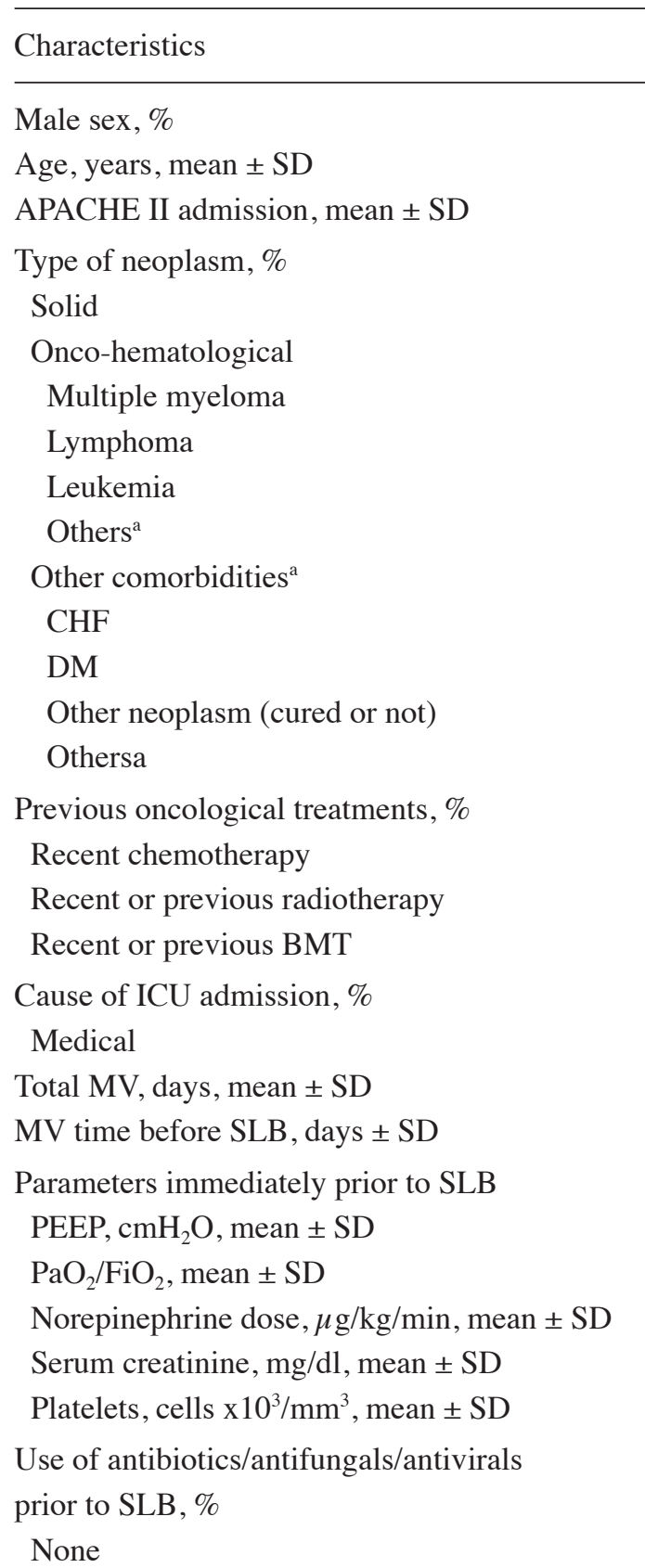

1-2 antibiotics/antifungals

$\geq 3$

Glycopeptides

Carbapenems

Amphotericin B or equinocandins

Trimethoprim-sulfamethoxazole

Gancyclovir

Polymyxin

Fluconazole

3rd - 4th generation cephalosporin

Aminoglycosides

Penicillin + penicillinase inhibitor

Acyclovir

Quinolones

Value

$70.6 \%$

$33.8 \pm 16.45$

$24.5 \pm 8.95$

0

100

0

41

53

6

29.5

6

12

6

12

88

12

12

100

$7.2 \pm 4.54$

$2.1 \pm 2.8$

$11.0 \pm 4.86$

$258.0 \pm 89.0$

$0.4 \pm 0.57$

$1.3 \pm 0.60$

$95.9 \pm 53.1$

0

12

88

88.2

70.5

52.9

41.2

35.3

17.6

17.6

12

12

6

6

0

$9.5 \pm 5.68$

Total ICU length of stay, days, mean \pm SD
Table I. Continued.

ICU mortality, \% 88.2

Death prior to histopathology result $\quad 35.3$

Hospital mortality, \%

88.2

ancludes non-malignant hematological disorders, such as bone marrow aplasia (1 patient). APACHE, acute physiology and chronic health evaluation score; CHF, congestive heart failure; DM, diabetes mellitus; BMT, bone marrow transplant; MV, mechanical ventilation; PEEP, positive end-expiratory pressure; SLB, surgical lung biopsy.

Table II. Biopsy histopathological results $(n=17)$.

\begin{tabular}{lc}
\hline No pathological findings & $\%^{\mathrm{a}}$ \\
\hline CMV & 17.6 \\
ARDS & 23.5 \\
Bacterial pneumonia & 17.6 \\
Alveolar hemorrhage & 17.6 \\
Pneumocystis jirovecii & 17.6 \\
Neoplastic infiltrate & 12 \\
PE & 6 \\
Unspecific infiltrate & 6 \\
Others & 0 \\
\hline
\end{tabular}

${ }^{a}$ The total exceeds $100 \%$ as some patients had more than one biopsy diagnosis. CMV, cytomegalovirus; ARDS, acute respiratory distress syndrome; PE, pulmonary embolism.

Table III. Complications associated with the procedure $(n=17)$.

\begin{tabular}{ll}
\hline Complications & Incidence, $\%$
\end{tabular}

Hemoptysis, minor

0

Hemoptysis, major

Pneumotorax/broncho-pleural fistula

0

Minor bleeding from the thorax drain ${ }^{\mathrm{a}}$

0

Major bleeding from the thorax drain ${ }^{\mathrm{b}}$

41

Need to re-operation

9

Worsening of hypoxemia/need for

0

increased ventilatory parameters

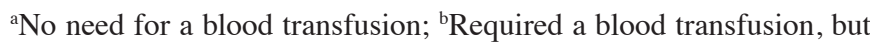
there was no need to re-operate.

Previous studies have had inconsistent findings in terms of etiology prevalence. Infection was usually reported as the most common cause of $\mathrm{ARpF}$ in oncohematological patients. Among infections, bacterial and mixed are the most common previously reported etiologies. Opportunistic infections-such as fungal, viral and $P$. jirovecii are also frequently described (up to $30 \%$ ). Non-infectious causes were less frequent, but very relevant management-wise-acute pulmonary edema (most incident non-infectious), alveolar hemorrhage, pulmonary 


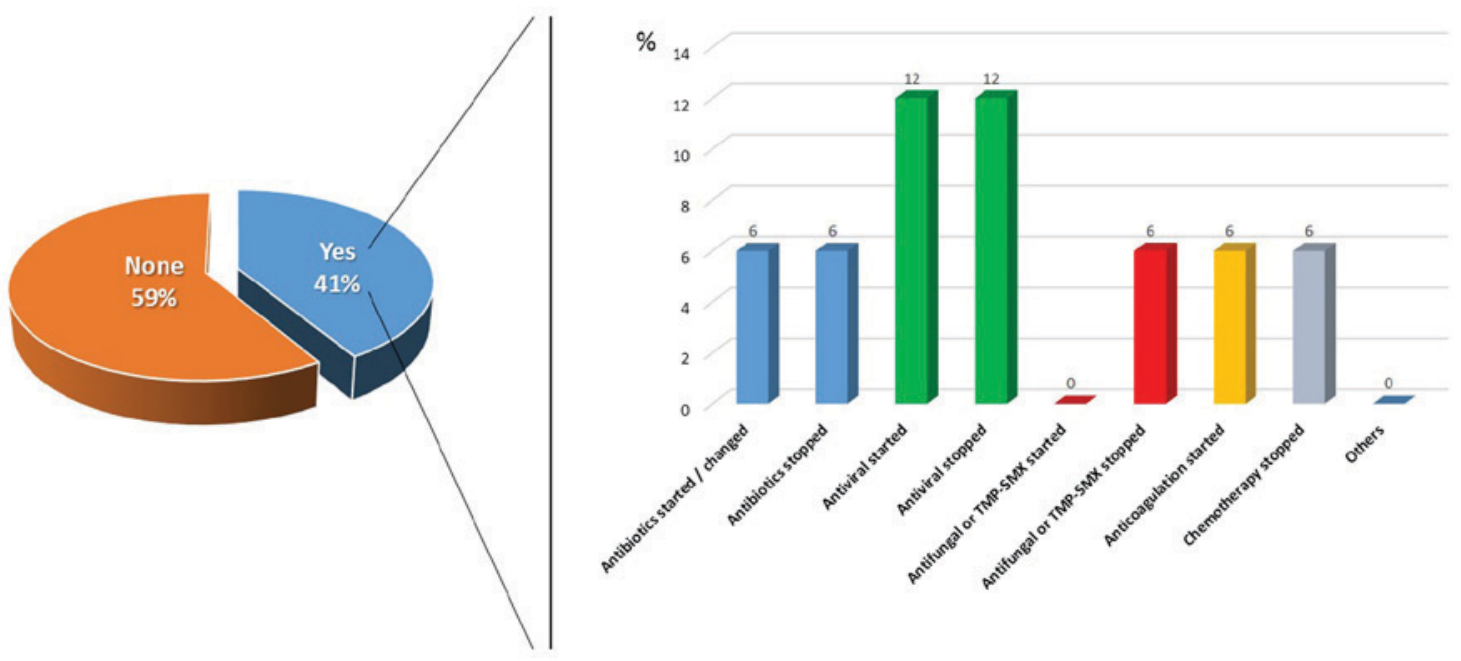

Figure 1. Therapeutic modifications following biopsy results (n=17). TMP-SMX, trimethoprim-sulfamethoxazole.

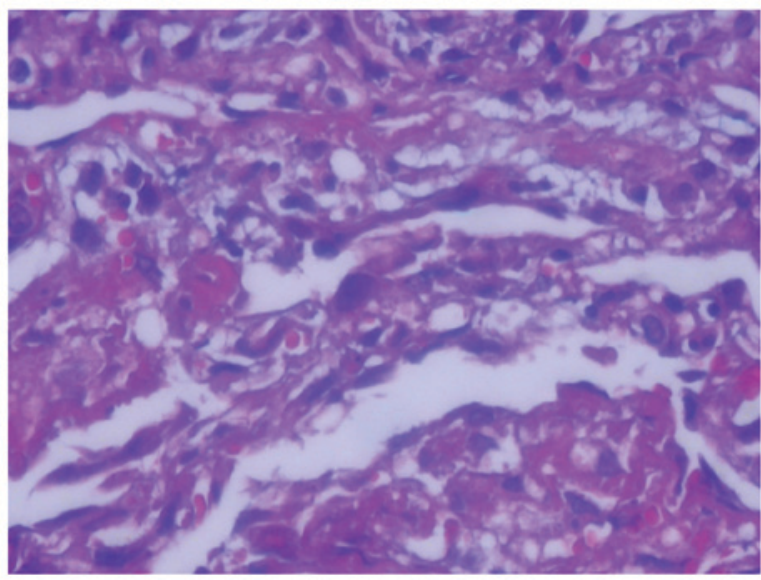

Figure 2. A 12 year-old female with lymphoma developing CMV pneumonitis. Biopsy slide OS-585554: Hematoxylin and eosin staining, magnification, $\mathrm{x} 400$. Pulmonary slide showing septs with lymphoid infiltrate, fibrinous material, with evidence in the center of the slide of a large endothelial cell with intense nuclear inclusion, rounded, condensed chromatin and poorly defined nuclear membrane, pressing the cytoplasm peripherally, characterizing a CMV infection. CMV, Cytomegalovirus.

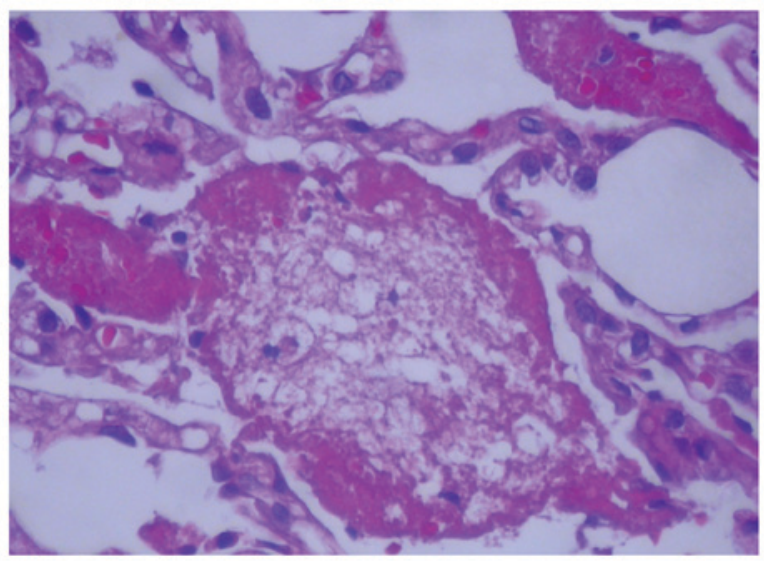

Figure 3. A 23 year-old male with acute leukemia developing Pneumocystis jirovecii Pneumonia. Biopsy slide OS-562073: Hematoxylin and eosin staining, magnification, $\mathrm{x} 400$. Pulmonary slide showing an alveolus partially hemorrhagic, filled by exudates of histiocytes with micro-vacuolated cytoplasm, cottonous and characteristics of Pneumocystis jirovecii infection.

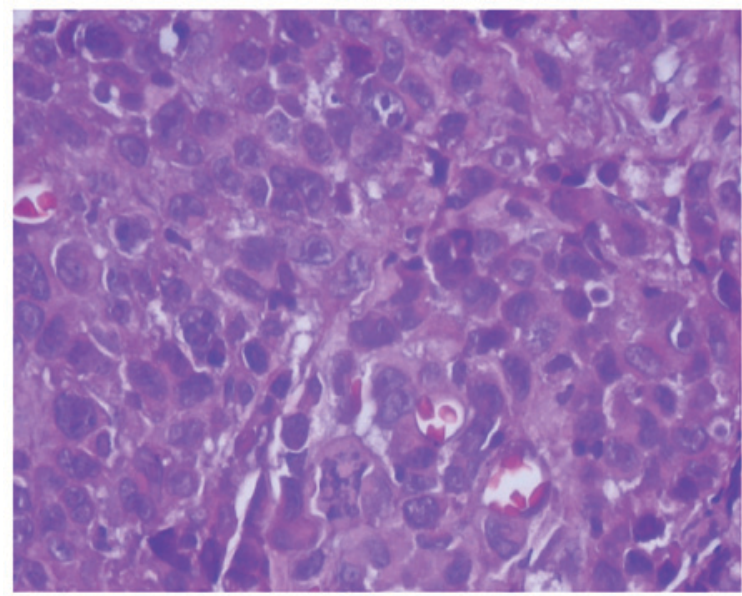

Figure 4. A 19 year-old male with Hodgkin's lymphoma, and lung infiltration by the disease. Biopsy slide OS-528700: Hematoxylin and eosin staining, magnification, $\mathrm{x} 400$. Pulmonary slide of an aggregate of atypical lymphoid cells, composed of large, bizarre, bi-nucleated nuclei that can be observed in the center of the figure. These cells also had central nucleoli and an evident, irregular, thickened nuclear membrane, with prominent and acidophilic cytoplasm, characteristic of Hodgkin's lymphoma, classical subtype.

embolism, TRALI, radiation pneumonitis, neoplastic infiltrate, Bronchiolitis obliterans/Organizing Pneumonia (BOOP) and chemotherapy-related adverse reactions $(10,11)$.

In our study, fungal infections were uncommon (17.6\%)-only 2 patients with $P$. jirovecii and none with Aspergillosis-, differently from other studies that have shown fungal infections as the most common and Aspergillosis as a frequent finding $(17,18,20)$. A possible reason for no Aspergillosis findings is that we had few (two) patients that had underwent BMT, a population with a high incidence of that infection (21). Regardless of that, the high prevalence of antifungals and Trimethoprim-Sulfamethoxazole (70.5 and $41.2 \%$, respectively) in our patients raised our attention as well. Other infectious causes, such as CMV and bacterial pneumonia, were found in our study and reported by previous literature with highly variable incidence. Unlike previous studies, we did not find any biopsy showing tuberculosis. 


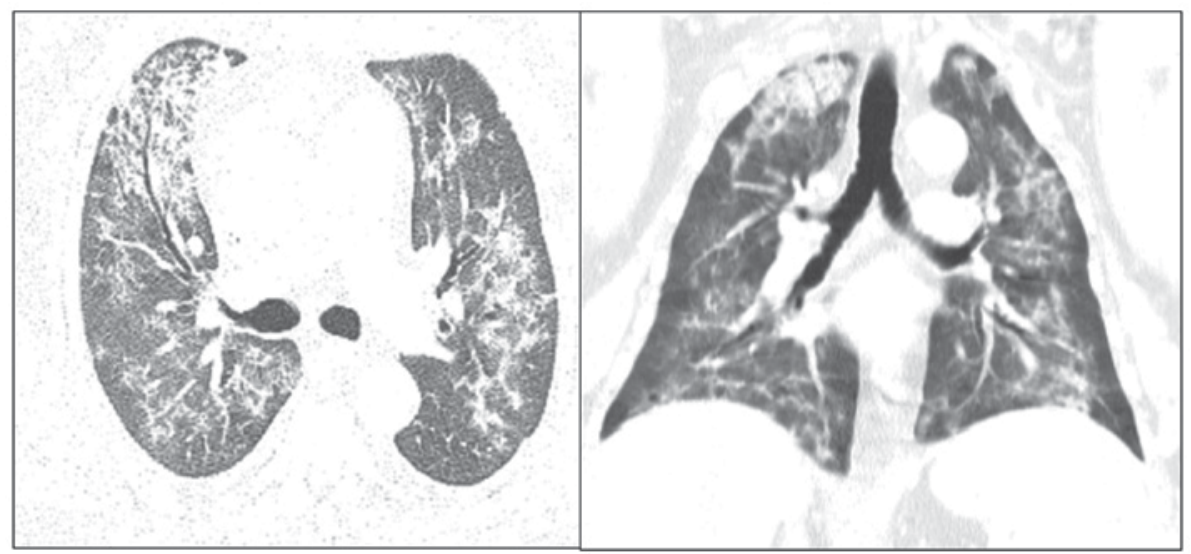

Figure 5. A 51 year-old male with multiple myeloma (post-autologous bone marrow transplant). Biopsy: Acute respiratory distress syndrome + alveolar hemorrhage. Chest computed tomography revealed heterogeneous bilateral pulmonary infiltrates, with a mosaic pattern.

Specific inflammatory findings, such as BOOP and granulomatous reactions were not found in our case series, even though they are reported as frequent in this patient group by the literature. Unspecific findings (ARDS and interstitial fibrosis) were found in $29.5 \%$ of biopsies, which is a similar prevalence to that of other studies (about 1/3). Alveolar hemorrhage was more prevalent (17.6\% - 3 biopsies) in our study than reported previously. Neoplastic infiltrates, present in 2 biopsies (12\%), was a frequent finding in the literature $(16-20,22,23)$.

Therapeutic modifications can be defined as starting or stopping of therapeutic strategies or adoption of exclusive palliative care (therapeutic limitation). The rate of therapeutic modification due to the biopsy results was $41 \%$, but, taking into account only the patients that were alive when the histopathological results were available, the rate was higher: $63.3 \%$. This data corroborates previous findings, in which therapeutic modification was performed in $40-70 \%$ of patients, with specific diagnosis associated with lower mortality $(16-20,22,23)$. Recently, studies on SLB in patients with pulmonary infiltrates and $\mathrm{ARpF}$, in a population with $50 \%$ of immunocompromised patients (but not onco-hematological), has shown the importance of SLB in the diagnosis of potentially reversible diseases, particularly through the usage of high dose corticoids, and, consequentially, with the potential to lower mortality rate (22).

Gay et al (16) have studied necropsies of oncological patients with lung infiltrates, adding diagnostic accuracy. Unfortunately, due to cultural and organizational problems (especially due to low societal acceptance of necropsies), we do not have access in our institution to necropsies done for scientific purposes; thus, we could not compare our SLB findings with those of post-mortem histopathological study. Also in relation to the transbronchial biopsy it was not possible to make a comparison with the data of the patients submitted to SLB, since the majority of the patients had high levels of PEEP and several had coagulation disorders/thrombocytopenia, for which surgical procedure was considered the safest option, both to contain possible bleeding and to prevent aerial fistulae.

However, it is necessary to outline that previous understanding of 'therapeutic modification' could underestimate the value of the SLB. Even in patients with unspecific findings, the information provided by the biopsy could have relevance in terms of avoiding further diagnostic and/or therapeutic interventions that could have been pursued if there was no histopathological confirmation. Particularly, it could lead to the complete suspension of aggressive and 'heroic' efforts, allowing palliative and end-of-life care to provide dignity to the patients and family members. A relevant fact is that about one third of the patients died before the histopathological result. This denotes the high severity of the patients (since the histopathological result in our institution is available between 36-96 $\mathrm{h}$ on average), but also possibly a delay in indicating and performing the procedure. In any case, the authors intentionally kept these patients, not only by the number of patients, but also to demonstrate a 'real life' situation (even if with non-ideal results).

In relation to SLB complications, in our study, no death occurred during the biopsy or that had been directly attributed to the procedure-for example, due to hemorrhagic shock or pneumothorax. Several authors had not reported, as well, attributable deaths to SLB, however, there has been literature reports of need for reoperation and increased time of MV $(16-19,22)$. There was no report in our study of pneumothorax and a very low incidence of major hemorrhages. No patient needed reoperation. Previous studies have shown $10-20 \%$ of complication in this population, and recently published study (in a non-oncological population) evidenced $12 \%$ of minor complications. Most commonly reported complications are bleeding and pneumothorax (including bronchopleural fistula), but there is also the chance of worsening respiratory failure and need to MV (in non-mechanically ventilated patients). In our study all patients were in MV already $(16-19,22)$. It should be emphasized, however, that due to the high mortality and intrinsic severity of the patients, possibly early or late unrecognized complications (such as worsening respiratory function or secondary infections) could have occurred, even if not recognized as secondary to the procedure itself.

Another question that warrants further analyzes is the impact of SLB in mortality. In our study, we found a mortality rate of almost $90 \%$ (no deaths were directly attributable to the procedure or its complications), higher than previously reported (18-45\%). Nevertheless, it is relevant to outline that in our study all the patients were in MV with high PEEP levels, which denotes higher severity and mortality $(16-20,22,23)$. 
Even though the authors could not correlate performing SLB and mortality, this was higher than previously reported (16-18). We believe that the high mortality we found could be secondary to several factors, such as: 1) Higher illness severity when compared to previous SLB population-which had a share of non-intubated patients. In our sample, all patients were intubated, most on high dose vasopressors and high PEEP levels. 2) The characteristics and eventual deficiencies in treatment and management. It has been shown, for example, that sepsis and ARDS patients in low to mid-income countries have worst outcomes than in high-income countries (24-26).

This study has several limitations that preclude the eventual generalization of its findings. It was a single center study, which makes the sample size (n) relatively small, but in line with the size of previous studies. This reduced size of the sample could justify the incidence of diseases (such as the lack of patients with tuberculosis) or even the low incidence of complications. Besides, for its local characteristics and possibly for a common limitation in developing countries, there was a paucity of less invasive procedures (such as BAL and serological tests in determined situations), and without performing necropsies in deceased patients (for eventual comparison), due to unavailability and/or high associated costs. However, this limitation puts it closer to the reality of developing countries. In addition to it, its design, a retrospective, observational, the reliability of data can be affected by the absence of previous uniformization of concepts to their registry, in addition to possibly time-related diagnosis and management changes (although the authors did not detect significant changes in the medical records evaluation). Also, it cannot establish the impact of the intervention (SLB) in morbidity and mortality, due to its design (observational, non-interventionist). Despite that, the goal of this study was to evaluate the 'real life' of an oncology ICU in a developing country, being appropriate for this objective.

Onco-hematological patients submitted to SLB in the ICU had an infectious process, mainly opportunistic, as the most common biopsy finding, followed by unspecific infiltrate. Therapeutic modification could be made in one third of the patients. However, hospital mortality in these patients was very high, possibly due to their illness severity. SLB helps the therapeutic management of these patients with few complications related to the intervention.

\section{Acknowledgements}

Not applicable.

\section{Funding}

No funding was received.

\section{Availability of data and materials}

The datasets used and/or analysed during the current study are available from the corresponding author on reasonable request.

\section{Author's contributions}

EMC and PADD designed the study, collected and analyzed the data, and wrote the manuscript. AGB, DAP, ADC and RCS analyzed the data, wrote the manuscript and reviewed the manuscript. All the authors read and approved the final manuscript.

\section{Ethical approval and consent to participate}

This study was conducted in accordance with the recommendations of Resolution 466/2012 of the Brazilian National Health Council. The project was approved by Universidade Estadual do Oeste do Paraná Committee on Ethics in Research involving human beings (Cascavel, Brazil). Due to the retrospective nature of the study the requirement for patients' written informed consent was waived.

\section{Patient consent for publication}

Not applicable.

\section{Competing interests}

The authors declare that they have no competing interests.

\section{References}

1. Ferlay J, Soerjomataram I, Ervik M, Dikshit R, Eser S, Mathers C,Rebelo M, Parkin DM, Forman D and Bray F (eds): GLOBOCAN 2012: Estimated Cancer Incidence, Mortality and Prevalence Worldwide in 2012 v1.0. Int Agency Res Cancer, Lyon, France, 2013.

2. Brenner H: Long-term survival rates of cancer patients achieved by the end of the 20th century: A period analysis. Lancet 360 : 1131-1135, 2002.

3. Angus DC, Barnato AE, Linde-Zwirble WT, Weissfeld LA, Watson RS, Rickert T and Rubenfeld GD; Robert Wood Johnson Foundation ICU End-Of-Life Peer Group: Use of intensive care at the end of life in the United States: An epidemiologic study. Crit Care Med 32: 638-43, 2004.

4. Schellongowski P, Sperr WR, Wohlfarth P, Knoebl P, Rabitsch W, Watzke HH and Staudinger T: Critically ill patients with cancer: Chances and limitations of intensive care medicine-a narrative review. ESMO Open 1: e000018, 2016.

5. Nazer L, Al-Shaer M and Hawari F: Drug utilization pattern and cost for the treatment of severe sepsis and septic shock in critically ill cancer patients. Intern J Clin Pharm 35: 1245-1250, 2013.

6. Groeger JS, Glassman J, Nierman DM, Wallace SK, Price K, Horak D and Landsberg D: Probability of mortality of critically ill cancer patients at $72 \mathrm{~h}$ of intensive care unit (ICU) management. Support Care Canc 11: 686-695, 2003.

7. Benoit DD, Vandewoude KH, Decruyenaere JM, Hoste EA and Colardyn FA: Outcome and early prognostic indicators in patients with a hematologic malignancy admitted to the intensive care unit for a life-threatening complication. Crit Care Med 31: 104-112, 2003

8. Pastores SM and Voigt LP: Acute respiratory failure in the patient with cancer: Diagnostic and management strategies. Crit Care Clin 26: 21-40, 2010.

9. Cooke CR: Economics of mechanical ventilation and respiratory failure. Crit Care Clin 28: 39-55, 2012

10. Vadde R and Pastores SM: Management of acute respiratory failure in patients with hematological malignancy. J Intens Care Med 31: 627-641, 2016.

11. AzoulayE,MokartD,LambertJ,Lemiale V,RabbatA,KouatchetA, Vincent F, Gruson D, Bruneel F, Epinette-Branche G, et al: Diagnostic strategy for hematology and oncology patients with acute respiratory failure: Randomized controlled trial. Am J Respir Crit Care Med 182: 1038-1046, 2010.

12. Azoulay E and Schlemmer B: Diagnostic strategy in cancer patients with acute respiratory failure. Intens Care Med 32: 808-822, 2006.

13. ARDS Definition Task Force; Ranieri VM, Rubenfeld GD, Thompson BT, Ferguson ND, Caldwell E, Fan E, Camporota L and Slutsky AS: Acute respiratory distress syndrome: The Berlin definition. JAMA 307: 2526-2533, 2012. 
14. Mehta RL, Kellum JA, Shah SV, Molitoris BA, Ronco C, Warnock DG and Levin A; Acute Kidney Injury Network: Acute Kidney Injury Network: Report of an initiative to improve outcomes in acute kidney injury. Crit Care 11: R31, 2007.

15. Singer M, Deutschman CS, Seymour CW, Shankar-Hari M, Annane D, Bauer M, Bellomo R, Bernard GR, Chiche JD, Coopersmith CM, et al: The third international consensus definitions for sepsis and septic shock (Sepsis-3). JAMA 315: 801-810, 2016.

16. Gay J, Lemiale V, Meignin V, Bron C, De Bazelaire C, Schnell D, Canet E, Seguin A and Azoulay E: Diagnostic contribution from pulmonary biopsies in hematology patients with acute respiratory failure from undetermined etiology. Minerva Anestesiol 79: 853-860, 2013

17. Zihlif M, Khanchandani G, Ahmed HP and Soubani AO: Surgical lung biopsy in patients with hematological malignancy or hematopoietic stem cell transplantation and unexplained pulmonary infiltrates: Improved outcome with specific diagnosis. Am J Hematol 78: 94-99, 2005.

18. White DA, Wong PW and Downey R: The utility of open lung biopsy in patients with hematologic malignancies. Am J Resp Crit Care Med 161: 723-729, 2000.

19. Snyder CL, Ramsay NK, Mcglave PB, Ferrell KL and Leonard AS: Diagnostic open-lung biopsy after bone marrow transplantation. J Pediatr Surg 25: 871-877, 1990.

20. Kramer MR, Berkman N, Mintz B, Godfrey S, Saute M and Amir G: The role of open lung biopsy in the management and outcome of patients with diffuse lung disease. Ann Thor Surg 65 : 198-202, 1998.
21. Hayes-Jordan A, Benaim E, Richardson S, Joglar J, Srivastava DK, Bowman L and Shochat SJ: Open lung biopsy in pediatric bone marrow transplant patients. J Pediatr Surg 37: 446-452, 2002.

22. Gerard L, Bidoul T, Castanares-Zapatero D, Wittebole X, Lacroix V, Froidure A, Hoton D and Laterre PF: Open lung biopsy in nonresolving acute respiratory distress syndrome commonly identifies corticosteroid-sensitive pathologies, associated with better outcome. Crit Care Med 46: 907-914, 2018.

23. Hilbert G, Gruson D, Vargas F, Valentino R, Gbikpi-Benissan G, Dupon M, Reiffers J and Cardinaud JP: Noninvasive ventilation in immunosuppressed patients with pulmonary infiltrates, fever, and acute respiratory failure. N Engl J Med 344: 481-487, 2001.

24. Rudd KE, Kissoon N, Limmathurotsakul D, Bory S, Mutahunga B, Seymour CW, Angus DC and West TE: The global burden of sepsis: Barriers and potential solutions. Crit Care 22: 232, 2018.

25. Becker JU, Theodosis C, Jacob ST, Wira CR and Groce NE: Surviving sepsis in low-income and middle-income countries: New directions for care and research. Lancet Infect Dis 9: 577-582, 2009.

26. Laffey J, Madotto F, Bellani G, Pham T, Fan E, Brochard L, Amin P, Arabi Y, Bajwa EK, Bruhn A, et al: Geo-economic variations in epidemiology, patterns of care, and outcomes in patients with acute respiratory distress syndrome: Insights from the LUNG SAFE prospective cohort study. Lancet Resp Med 5: 627-638, 2017. 